UCLA/98/TEP/14

hep-lat/9806030

\title{
Absence of confinement in the absence of vortices
}

\author{
Tamás G. Kovács円 \\ Department of Physics, University of Colorado, Boulder CO 80309-390 \\ e-mail: kovacs@eotvos.Colorado.EDU \\ and \\ E. T. Tomboulis? \\ Department of Physics, UCLA, Los Angeles, CA 90095-1547 \\ e-mail: tombouli@physics.ucla.edu
}

\begin{abstract}
We consider the Wilson loop expectation in $S U(2)$ lattice gauge theory in the presence of constraints. The constraints eliminate from the functional measure gauge field configurations whose physical interpretation is that of thick center vortices linking with the loop. We give a simple proof that, for dimension $d \geq 3$, the so constrained Wilson loop follows perimeter law, i.e. non-confining behavior, at weak coupling (low temperature). Thus the presence of vortex configurations is a necessary condition for confinement.
\end{abstract}

\footnotetext{
${ }^{1}$ Research supported by DOE grant DE-FG02-92ER-40672

${ }^{2}$ Research supported by NSF grant nsf-phy 9531023
} 


\section{Introduction}

The precise physical mechanism(s) by which $S U(N)$ gauge theories in their critical dimension apparently avoid a phase transition and thus remain in a confining phase for arbitrarily weak coupling is currently receiving renewed attention (see e.g. [1]). The proposal that extended thick vortices are the configurations responsible for this behavior has been strongly supported by recent numerical simulations in the case of $S U(2)$ [2], [3]. (We refer to [2] for a recent discussion of the underlying heuristic physical picture, as well as earlier references to these ideas.) The string tension of the full Wilson loop at weak coupling is found in these simulations to be fully reproduced solely by the contribution of thick vortices. In this paper we give a simple proof of the converse statement, also seen in the simulations. We consider the Wilson loop in the presence of constraints eliminating field configurations which, in physical terms, represent thick vortices linking with the loop. We then show that the Wilson loop exhibits non-confining (perimeter law) behavior at weak coupling.

An earlier related result was obtained some time ago in [4]. There it is proven that, in the presence of constraints eliminating vortices winding around the (periodic) lattice, the electric-flux free energy order parameter exhibits nonconfining behavior at large $\beta$. Asymptotically large Wilson loops are indeed expected to be physically equivalent to the electric-flux order parameter. The latter, however, has been proven rigorously [5] only to form an upper bound on the Wilson loop. Thus it only provides a sufficient criterion for confinement: confining behavior of the electric-flux free energy implies confining behavior for the Wilson loop, but not the converse. At any rate, the electric-flux is an order parameter that refers to the entire lattice. It is clearly important to obtain a statement concerning the effect of the constraints on the Wilson loop itself, since it directly represents the actual potential between two external sources (quarks), as the lattice is taken to the thermodynamic limit.

The distinction between 'thick', 'thin' and 'hybrid' vortices is crucial to the physical picture of the action of vortices at small gauge coupling (large $\beta$ ). An appropriate framework for these considerations is obtained by expressing the $S U(N)$ lattice gauge theory in terms of new separate $Z(N)$ and $S U(N) / Z(N)$ variables [6], [0]. We review this formulation in section 2 , where we also consider a variety of alternative lattice actions for which we obtain our result. We also introduce the appropriate constraints for eliminating linking thick and hybrid vortices. The proof that the so constrained Wilson loop follows perimeter law is given in section 3. We explicitly consider only the case of $S U(2)$ which exhibits all the relevant physical features of the general $N$ case.

Notation. We work on a simple hypercubic lattice $\Lambda \subset \mathbf{Z}^{d}$ of size $L_{\mu}=2^{N_{\mu}}$, integer $N_{\mu}$, and lattice coordinates integer $n^{\mu}, \mu=1, \ldots, d$. Elementary cells $c^{r}$, $(r=0,1, \ldots, d)$, in $\Lambda$ will be denoted more explicitly as $s$ (sites), $b$ (bonds), $p$ (plaquettes), $c$ (3-cubes), etc. Each $r$-cell is assigned an orientation; $-c^{r}$ then denotes the oppositely oriented cell. The number of cells in a collection $S$ of $r$-cells will be denoted by $|S|$. The oriented $r$-cells are the generators of the integer $r$-chains. There 
is a natural inner product on the cell complex defined by: $\left(c^{r}, c^{q}\right)=1$ if $r=q$, and 0 otherwise. If then $\partial: c^{r} \rightarrow c^{r-1}$ is the boundary operator on $r$-chains, the coboundary operator $\partial^{*}$ is defined by:

$$
\left(\partial^{*} c^{r}, c^{r+1}\right)=\left(c^{r}, \partial c^{r+1}\right) \quad .
$$

Thus the coboundary of an $r$-cell $c^{r}$ is the $(r+1)$-chain formed of those $(r+1)$-cells (taken with appropriate orientation) whose boundaries contain $c^{r}$, i.e. $\pm c^{r+1} \in \partial^{*} c^{r}$ iff $\pm c^{r} \in \partial c^{r+1}$. By linearity this extends to arbitrary $r$-chains.

We employ the standard formalism of lattice gauge theory. With each bond we associate a copy $G_{b}$ of the gauge group $G$. The gauge field $U_{b}$ is an element of $G_{b}$, with the assignment $U_{-b}=U_{b}^{-1}$. The configuration space is then $\Omega_{\Lambda}=\otimes_{b \in \Lambda} G_{b}$, and a field configuration $U_{\Lambda}=\left\{U_{b}\right\}_{b \in \Lambda}$ is an element of $\Omega_{\Lambda}$. In the following we do not distinguish explicitly between the abstract group element $U_{b}$ and its matrix representation which, unless otherwise indicated, will always be the fundamental representation.

We will introduce various lattice variables taking values in the center of the group $G$. If $K$ is an abelian group, multiplicatively written, a $K$-valued $r$-form $\alpha$ is the map: $\alpha: c^{r} \rightarrow \alpha\left[c^{r}\right] \in K$, with $\alpha\left[-c^{r}\right]=\alpha\left[c^{r}\right]^{-1}$. The exterior difference operator is then defined by

$$
(d \alpha)\left[c^{r+1}\right]=\prod_{c^{r} \in \partial c^{r+1}} \alpha\left[c^{r}\right]
$$

Given any set $Q$ of $c^{k}$ cells, we generally employ the shorthand notation

$$
\alpha[Q]=\alpha\left[\prod_{k \in Q} c^{k}\right]=\prod_{k \in Q} \alpha\left[c^{k}\right] \equiv \alpha_{Q}
$$

Thus, e.g., for a 1-form $\gamma$ we write $\gamma_{b},(d \gamma)_{p}$, and so on. In this paper $G=S U(2)$.

\section{Actions and constraints}

The Euclidean functional measure of the standard lattice $S U(2)$ theory is given by:

$$
d \mu_{\Lambda}(U)=Z_{\Lambda}^{-1} \prod_{b \in \Lambda} d U_{b} \exp \left(\sum_{p} A_{p}\left(U_{p}\right)\right)
$$

where $d U_{b}$ denotes normalized Haar measure on $S U(2)$, and $A_{p}$ the plaquette action which is a function of $U_{p}=\prod_{b \in \partial p} U_{b}$, the product of bond variables $U_{b}$ around the plaquette $p$. The partition function $Z_{\Lambda}$ is defined by $\int d \mu_{\Lambda}(U)=1$, and for any observable $F(U)$, i.e. (complex-valued) function on $\Omega_{\Lambda}$

$$
\langle F(U)\rangle=\int d \mu_{\Lambda}(U) F(U)
$$


The usual minimal plaquette action is the Wilson action

$$
A_{p}\left(U_{p}\right)=\beta \operatorname{tr} U_{p}
$$

which is a special case of the action

$$
A_{p}\left(U_{p}\right)=\beta \operatorname{tr} U_{p}+\lambda \operatorname{sign} \operatorname{tr} U_{p} .
$$

(2.4) extrapolates between the Wilson action $(\lambda=0)$ and the 'positive plaquette model' action $(\lambda \rightarrow \infty)$. Another action we consider in this paper is

$$
A_{p}\left(U_{p}\right)=\beta \operatorname{tr} U_{p}+\ln \left(\theta\left(\left|\operatorname{tr} U_{p}\right|-k\right)\right),
$$

where $\theta(x)=1$ if $x>0,0$ if $x<0$, and $0<k<2$; in particular, $k$ a constant, or any function of $\beta$ such that $k \beta \rightarrow \infty$ as $\beta \rightarrow \infty$, e.g. $k(\beta)=\beta^{-1 / 2}$. All these actions have, of course, the same naive continuum limit. More generally, they are all physically equivalent for sufficiently large $\beta$, where each plaquette becomes highly peaked around the perturbative vacuum $\operatorname{tr} U_{p} \rightarrow 1$. Use of such alternative actions provides a check of the requirement that long distance physics should not depend on short distance details such as the precise form of the latticized action. We will first prove our result working with the action (2.5) since, as it turns out, it allows a rather simpler proof of the result. We will then obtain the result for the more standard, but in fact physically equivalent, action (2.4).

To formulate our argument we rewrite the $S U(2)$ theory (2.1) in the $S O(3) \times$ $Z(2)$ form [6], [7]. Consider the configuration space $\Omega_{\Lambda}$ split into equivalence classes, each class the coset bond variable configuration $\hat{U}_{\Lambda}=\left\{\hat{U}_{b}\right\}_{b \in \Lambda}, \hat{U}_{b} \in S U(2) / Z(2) \sim$ $S O(3)$. Thus two configurations $U_{\Lambda} U_{\Lambda}^{\prime} \in \Omega_{\Lambda}$ are representatives of the same coset configuration $\hat{U}_{\Lambda}$ iff one has $U_{b}^{\prime}=U_{b} \gamma_{b}$, for some $\gamma \in Z(2), \forall b \in \Lambda$. Let $\eta_{p}(U) \equiv$ $\operatorname{sgn} \operatorname{tr} U_{p}$. Then

$$
(d \eta)_{c}(\hat{U})=\prod_{p \in \partial c} \eta_{p}
$$

depends, as indicated, only on the coset configuration, since it is invariant under $U_{b} \rightarrow$ $U_{b} \gamma_{b}, \gamma \in Z(2)$. Let $\sigma$ be a $Z(2)$-valued 2 -form on $\Lambda$. Adopt periodic boundary conditions (b.c.), and let $\left\{S^{\alpha}\right\}, \alpha=1, \ldots,\left(\begin{array}{l}d \\ 2\end{array}\right)$, be a set of 2-dimensional nonbounding closed surfaces forming a 2-cycle basis on $\Lambda=T^{d}\left(N_{1}, \ldots, N_{d}\right)$. Then the $S U(2)$ theory (2.1) - (2.2) can be expressed in the $S O(3) \times Z(2)$ form given by:

$$
\begin{gathered}
d \mu_{\Lambda}(U, \sigma)=Z_{\Lambda}^{-1} \prod_{b \in \Lambda} d U_{b} \prod_{p \in \Lambda} d \sigma_{p} \prod_{c \in \Lambda} \delta\left((d \eta)_{c}(d \sigma)_{c}\right) \prod_{\alpha} \delta\left(\eta\left[S^{\alpha}\right] \sigma\left[S^{\alpha}\right]\right) \\
\exp \left(\sum_{p \in \Lambda} A_{p}\left(\left|\operatorname{tr} U_{p}\right|, \sigma_{p}\right)\right) .
\end{gathered}
$$

In (2.7) $\int d \sigma_{p}(\cdots) \equiv \frac{1}{2} \sum_{\sigma_{p}= \pm 1}(\cdots)$ denotes Haar measure over $Z(2)$, and

$$
\delta(\alpha) \equiv \int d \tau \chi_{\tau}(\alpha)=\frac{1}{2}[1+\alpha]
$$


defines the delta-function on the group $Z(2)$. Here $\chi_{\tau}(\alpha)=\alpha$ if $\tau=-1$, and 1 otherwise, are the characters of $Z(2)$. The partition function $Z_{\Lambda}$ is defined by $\int d \mu_{\Lambda}(\hat{U}, \sigma)=1$. The plaquette action is given by

$$
A_{p}\left(\left|\operatorname{tr} U_{p}\right|, \sigma_{p}\right)=\beta\left|\operatorname{tr} U_{p}\right| \sigma_{p}+\lambda \sigma_{p}
$$

and

$$
A_{p}\left(\left|\operatorname{tr} U_{p}\right|, \sigma_{p}\right)=\beta\left|\operatorname{tr} U_{p}\right| \sigma_{p}+\ln \left(\theta\left(\left|\operatorname{tr} U_{p}\right|-k\right)\right)
$$

corresponding to (2.4) and (2.5), respectively. (The result for the Wilson action is, of course, given by (2.9) with $\lambda=0$.) (2.7) is easily seen to be independent of the choice of basis $\left\{S^{\alpha}\right\}$. Note that (2.7) is indeed a measure on the coset configuration space since it is invariant under $U_{b} \rightarrow U_{b} \gamma_{b}$, for any $Z(2)$-valued 1-form $\gamma$. If $\hat{F}(\hat{U}, \sigma)$ expresses a gauge invariant observable $F(U)$ in the new variables, the expectation (2.2) then satisfies

$$
\langle F(U)\rangle=\int d \mu_{\Lambda}(U, \sigma) \hat{F}(\hat{U}, \sigma)
$$

Free b.c. on $U_{\partial \Lambda}$ results into (2.7) without the product of $\delta$-functions over 2cycles, and free b.c. on $\hat{U}_{\partial \Lambda}, \sigma_{\partial \Lambda}$. By selective omission of factors in this product, one may consider any mixture of free and periodic b.c. (2.7) may be straightforwardly generalized to accommodate other useful types of b.c., [3 and indeed applied to $\Lambda$ of any torsionless homology.

We are interested in the expectation of a Wilson loop subject to constraints whose physical effect is the elimination of its interaction with those vortices that are not energetically directly suppressed by the plaquette action at large $\beta$. These are extended vortices in the $\hat{U}_{\Lambda}$ configurations, 'thick' vortices. 7 Thin vortices, vortices in the $\sigma_{\Lambda}$ configurations, are directly suppressed by action cost proportional to their length. There are also hybrid vortices formed by the joining of 'punctured' thick and thin vortices along their common coboundary which represents a magnetic current 'loop', i.e. a coclosed set of 3 -cubes $\mathcal{L}$ with $(d \eta)_{c}=(d \sigma)_{c}=-1, \forall c \in \mathcal{L}$. Such hybrids with a minimal short thin section but extended thick section can also affect long distance behaviour at large $\beta$. We refer to $([2])$, and $([4]),([6]),([\mathbb{8}])$, for discussion of the physical interpretation of the constraints we now introduce.

We consider the constrained Wilson loop expectation:

$$
W[C]=\left\langle\operatorname{tr} U[C] \theta\left[\operatorname{tr} U[C] \eta_{S}\right]\right\rangle
$$

\footnotetext{
${ }^{3}$ In particular, 'twisted' periodic b.c. on the torus given by action $\tau^{\alpha} A_{p}\left(U_{p}\right), \tau_{\alpha} \in Z(2)$, for $p \in S_{\alpha}^{*}$, where $\left\{S_{\alpha}^{*}\right\}$ a 2-cocycle basis, $\left(S_{\alpha}^{*}, S^{\beta}\right)=\delta_{\alpha}^{\beta}$, result into 2.7 with periodic b.c. and the substitution $\delta\left(\tau_{\alpha} \eta\left[S^{\alpha}\right] \sigma\left[S^{\alpha}\right]\right)$ for each factor in the product over 2-cycles. Note that b.c. defined by summation over the twist $\tau_{\alpha}$ eliminate the corresponding $\delta$-function factor. Such b.c. enter in the definition of the magnetic- and electric-flux free energy order parameters.

${ }^{4}$ In the continuum extrapolation they are configurations topologically characterized by nontrivial element(s) of $\pi_{1}(S U(2) / Z(2))=Z(2)\left(\pi_{1}(S U(N) / Z(N))=Z(N)\right.$ for general $\left.N\right)$.
} 
where now $\langle-\rangle$ denotes expectations in the restriction of the measure (2.1) to

$$
d \mu_{\Lambda}(U)=Z_{\Lambda}^{-1} d \nu_{\Lambda}(U) \exp \left(\sum_{p \in \Lambda} A_{p}\left(U_{p}\right)\right),
$$

with

$$
d \nu_{\Lambda}(U)=\prod_{b \in \Lambda} d U_{b} \prod_{c \in \Lambda} \delta\left((d \eta)_{c}\right) \prod_{\alpha} \delta\left(\eta\left[S^{\alpha}\right]\right)
$$

and periodic b.c. $\left(Z_{\Lambda}\right.$ defined by $\left.\langle 1\rangle=1\right)$. The constraint [6]

$$
\prod_{c} \delta\left((d \eta)_{c}\right)
$$

in the measure (2.13) eliminates all monopoles, and hence all hybrid vortices in the theory. The factor

$$
\theta\left[\operatorname{tr} U[C] \eta_{S}\right]
$$

where $\eta_{S} \equiv \prod_{p \in S} \eta_{p}$, which modifies the Wilson loop operator $\operatorname{tr} U[C]$ in (2.12), eliminates precisely all thick (unpunctured) vortices (while still allowing all thin vortices) linked with the loop [4], [2]. Here $S$ is any surface bounded by the loop: $C=\partial S$. The expectation (2.12) is easily seen to be independent of the choice of $S$. The remaining constraint

$$
\prod_{\alpha} \delta\left(\eta\left[S^{\alpha}\right]\right)
$$

in (2.13) is physically inessential, f and only included for technical convenience in adopting periodic b.c. It can be eliminated by adopting free b.c. or twisted plus untwisted periodic b.c. (cp. footnote 3).

We pass then to $S O(3) \times Z(2)$ variables in which (2.12) assumes the form

$$
\begin{array}{r}
W[C]=Z_{\Lambda}^{-1} \int d \nu_{\Lambda}(U) \prod_{p \in \Lambda} d \sigma_{p} \prod_{c \in \Lambda} \delta\left((d \eta)_{c}(d \sigma)_{c}\right) \prod_{\alpha} \delta\left(\eta\left[S^{\alpha}\right] \sigma\left[S^{\alpha}\right]\right) \\
\cdot \exp \left(\sum_{p} A_{p}\left(\left|\operatorname{tr} U_{p}\right|, \sigma_{p}\right)\right) \operatorname{tr} U[C] \eta_{S} \sigma_{S} \theta\left[\operatorname{tr} U[C] \eta_{S}\right] \\
=Z_{\Lambda}^{-1} \int d \nu_{\Lambda}(U) \prod_{b \in \Lambda} d \gamma_{b} \exp \left(\sum_{p} A_{p}\left(\left|\operatorname{tr} U_{p}\right|,(d \gamma)_{p}\right)\right) \\
\theta\left[\operatorname{tr} U[C] \eta_{S}\right] \operatorname{tr} U[C] \eta_{S} \gamma[C] .
\end{array}
$$

where $\gamma$ a $Z(2)$-valued 1-form. The second equality is obtained by solving the constraints on $\sigma_{\Lambda}$ that result from the restriction to (2.14):

$$
\begin{aligned}
(d \sigma)_{c}=1, \forall c, \quad \delta\left(\sigma\left[S^{\alpha}\right]\right)=1, \forall \alpha \Longrightarrow \sigma_{p} & =\prod_{b \in \partial p} \gamma_{b}=(d \gamma)_{p} \\
\sigma_{S} & \equiv \prod_{p \in S} \sigma_{p}=\prod_{b \in C} \gamma_{b}=\gamma[C] .
\end{aligned}
$$

\footnotetext{
${ }^{5}$ It is in fact automatically enforced as $|\Lambda| \rightarrow \infty$ at large $\beta$ because of the energetic suppression of thin vortices winding completely around the lattice. This can be seen clearly from (2.16) below.
} 
Now,

$$
\theta\left[\operatorname{tr} U[C] \eta_{S}\right] \operatorname{tr} U[C] \eta_{S}=\theta\left[\operatorname{tr} U[C] \eta_{S}\right]|\operatorname{tr} U[C]|
$$

so we can write $(2.12)$ in the form

$$
W[C]=\left\langle\theta\left[\operatorname{tr} U[C] \eta_{S}\right]|\operatorname{tr} U[C]| \gamma[C]\right\rangle_{S O(3) \otimes Z(2)}
$$

where

$$
\langle-\rangle_{S O(3) \otimes Z(2)}=\int d \mu_{\Lambda}(U, \gamma)(-)
$$

with

$$
d \mu_{\Lambda}(U, \gamma) \equiv Z_{\Lambda}^{-1} d \hat{\nu}_{\Lambda}(U) \prod_{b \in \Lambda} d \gamma_{b} \exp \left(\sum_{p \in \Lambda} K_{p}(U)(d \gamma)_{p}\right)
$$

and periodic b.c. $d \hat{\nu}_{\Lambda}(U)$ and $K_{p}(U)=K_{p}(\hat{U})$ are defined as

$$
\begin{aligned}
d \hat{\nu}_{\Lambda}(U) & \equiv d \nu_{\Lambda}(U) \\
K_{p}(U) & \equiv \beta\left|\operatorname{tr} U_{p}\right|+\lambda
\end{aligned}
$$

for the action (2.9), and

$$
\begin{aligned}
d \hat{\nu}_{\Lambda}(U) & \equiv d \nu_{\Lambda}(U) \prod_{p \in \Lambda} \theta\left(\left|\operatorname{tr} U_{p}\right|-k\right) \\
K_{p}(U) & \equiv \beta\left|\operatorname{tr} U_{p}\right|
\end{aligned}
$$

for the action (2.10). It is easily verified that (2.22) is a reflection positive measure in $(d-1)$-dimensional planes with sites.

Our task in the following is to bound the constrained Wilson loop expectation $(2.20)$ from below.

\section{Absence of confinement}

We formulate our main result as

Theorem 3.1 For sufficiently large $\beta$, and dimension $d \geq 3$ the constrained Wilson loop expectation (2.19), or, equivalently, 2.20), exhibits perimeter law, i.e. there exist constants $\alpha, \alpha_{1}(d), \alpha_{2}(d)$ such that

$$
W[C] \geq \alpha \exp \left(-\alpha_{2} e^{-\alpha_{1} \beta}|C|\right) \quad .
$$


From now on all expectation signs are meant in the measure (2.22), and, for brevity, we drop the label $S O(3) \otimes Z(2)$.

Choose the loop $C$ so that it is bisected into two equal pieces by a $(d-1)$-dim plane with sites. Then reflection positivity $(\mathrm{RP})$ of the measure (2.22) implies

$$
\left\langle\operatorname{tr} U[C] \eta_{S} \gamma[C]\right\rangle \geq 0
$$

(Without loss of generality, the surface $S$ may always be assumed to be also reflection symmetric in the hyperplane bisecting $C$.) Inserting

$$
1=\theta\left[\operatorname{tr} U[C] \eta_{S}\right]+\theta\left[-\operatorname{tr} U[C] \eta_{S}\right]
$$

in $(3.2)$ we then have

$$
\begin{aligned}
0 & \leq\left\langle\theta\left[\operatorname{tr} U[C] \eta_{S}\right] \operatorname{tr} U[C] \eta_{S} \gamma[C]\right\rangle+\left\langle\theta\left[-\operatorname{tr} U[C] \eta_{S}\right] \operatorname{tr} U[C] \eta_{S} \gamma[C]\right\rangle \\
& =\left\langle\theta\left[\operatorname{tr} U[C] \eta_{S}\right]|\operatorname{tr} U[C]| \gamma[C]\right\rangle-\left\langle\theta\left[-\operatorname{tr} U[C] \eta_{S}\right]|\operatorname{tr} U[C]| \gamma[C]\right\rangle
\end{aligned}
$$

On the other hand

$$
\langle|\operatorname{tr} U[C]| \gamma[C]\rangle=\left\langle\theta\left[\operatorname{tr} U[C] \eta_{S}\right]|\operatorname{tr} U[C]| \gamma[C]\right\rangle+\left\langle\theta\left[-\operatorname{tr} U[C] \eta_{S}\right]|\operatorname{tr} U[C]| \gamma[C]\right\rangle .
$$

Adding (3.3) and (3.4) we have

$$
\left\langle\theta\left[\operatorname{tr} U[C] \eta_{S}\right]|\operatorname{tr} U[C]| \gamma[C]\right\rangle \geq \frac{1}{2}\langle|\operatorname{tr} U[C]| \gamma[C]\rangle
$$

Hence from (2.20) we obtain:

$$
W[C] \geq \frac{1}{2}\langle|\operatorname{tr} U[C]| \gamma[C]\rangle
$$

Now

$$
\begin{aligned}
& \frac{1}{2}\langle|\operatorname{tr} U[C]| \gamma[C]\rangle= Z_{\Lambda}^{-1} \int d \hat{\nu}_{\Lambda}(U) \frac{|\operatorname{tr} U[C]|}{2} \\
& \cdot \int \prod_{b \in \Lambda} d \gamma_{b} \gamma[C] \exp \left(\sum_{p \in \Lambda} K_{p}(U)(d \gamma)_{p}\right) \\
&=\int d \hat{\mu}_{\Lambda}(U) \frac{|\operatorname{tr} U[C]|}{2}\langle\gamma[C]\rangle_{Z(2)}\left(\left\{K_{p}(U)\right\}\right),
\end{aligned}
$$

where

$$
d \hat{\mu}_{\Lambda}(U) \equiv Z_{\Lambda}^{-1} d \hat{\nu}(U) Z_{Z(2), \Lambda}\left(\left\{K_{p}(U)\right\}\right)
$$

and

$$
\langle-\rangle_{Z(2)}\left(\left\{K_{p}\right\}\right)=Z_{Z(2), \Lambda}^{-1}\left(\left\{K_{p}\right\}\right) \int \prod_{b \in \Lambda} d \gamma_{b}(-) \exp \left(\sum_{p \in \Lambda} K_{p} \gamma_{p}\right)
$$


denotes expectations in the $Z(2)$ lattice gauge theory with plaquette couplings $K_{p}$, and partition function $Z_{Z(2), \Lambda}$ defined by $\langle 1\rangle_{Z(2)}\left(\left\{K_{p}\right\}\right)=1$. In (3.7) we have the $U$-dependent couplings $K_{p}(U) \geq 0$ given by (2.23), (2.24). By the Griffiths inequalities [9] applied to (3.9):

$$
\langle\gamma[C]\rangle_{Z(2)}\left(\left\{K_{p}(U)\right\}\right) \geq 0
$$

whereas

$$
\frac{1}{2}|\operatorname{tr} U[C]| \geq \frac{1}{4}|\operatorname{tr} U[C]|^{2}=\frac{1}{4}\left|\chi_{F}(U[C])\right|^{2}=\frac{1}{4}\left[\chi_{A}(U[C])+1\right]
$$

where $\chi_{F}$ and $\chi_{A}$ denote the $S U(2)$ characters in the fundamental and adjoint representation, respectively. Combining (3.10), (3.11) with (3.7) then gives

$$
\begin{aligned}
\frac{1}{2}\langle|\operatorname{tr} U[C]| \gamma[C]\rangle \geq & \frac{1}{4} \int d \hat{\mu}_{\Lambda}(U)\langle\gamma[C]\rangle_{Z(2)}\left(\left\{K_{p}(U)\right\}\right) \\
& +\frac{1}{4} \int d \mu_{\Lambda}(U, \gamma) \chi_{A}(U[C]) \gamma[C]
\end{aligned}
$$

Now by RP the 2nd term on the r.h.s. in (3.12) is non-negative. So, from (3.6), we obtain:

$$
W[C] \geq \frac{1}{4} \int d \hat{\mu}_{\Lambda}(U)\langle\gamma[C]\rangle_{Z(2)}\left(\left\{K_{p}(U)\right\}\right)
$$

Action (2.10). Completing the proof of the result in the case of the action (2.10) is now straightforward. Applying again Griffiths inequalities, one has

$$
\langle\gamma[C]\rangle_{Z(2)}\left(\left\{K_{p}\right\}\right) \geq\langle\gamma[C]\rangle_{Z(2)}\left(\left\{K_{p}^{\prime}\right\}\right), \quad K_{p} \geq K_{p}^{\prime}, \forall p
$$

With the measure given by (2.22), (2.24), (3.8), use of (3.14) in (3.13) gives

$$
W[C] \geq \frac{1}{4}\langle\gamma[C]\rangle_{Z(2)}(k \beta) .
$$

The r.h.s. is the loop expectation in the $Z(2)$ gauge theory with $K_{p}=k \beta, \forall p$.

It is a well-known result that, for sufficiently large $\beta$ and $d \geq 3$ :

$$
\langle\gamma[C]\rangle_{Z(2)}(\beta) \geq \text { Const. } \exp (-\rho(\beta)|C|),
$$

i.e. the expectation exhibits perimeter-law. A rigorous proof (see e.g. Thm. 3.17 in [10], [11]) is by standard polymer expansion either as low-temperature expansion in 'contours', 5 or, after a duality transformation, as high-temperature expansion. Hence, (3.15) gives perimeter-law lower bound on $W[C]$ for all $\beta$ such that $k \beta$ large enough. This conclude the proof of the theorem in the case of the action (2.10).

Action (2.9). A coclosed set of plaquettes which cannot be decomposed into two disjoint coclosed sets will be called a contour. (Two plaquettes are defined to be disjoint if they share no link.) Given a configuration $\left\{\gamma_{b}\right\}_{b \in \Lambda}$, the set of plaquettes on

\footnotetext{
${ }^{6} \mathrm{~A}$ special case of the expansion we use in treating the action (2.9) below.
} 
which $(d \gamma)_{p}=-1$ is a coclosed set $\left((d-2)\right.$-dim closed set on the dual lattice $\left.\Lambda^{*}\right)$. Such a coclosed set $\Gamma$ can be uniquely decomposed into a disjoint contours $\zeta_{1}, \ldots, \zeta_{|\Gamma|}$. Each such contour then is the site of a thin vortex. We expand the expectation $\langle\gamma[C]\rangle_{Z(2)}\left(\left\{K_{p}(U)\right\}\right)$ in 3.13$)$ in a contour expansion. The partition function in the denominator is expanded in the form:

$$
Z_{Z(2), \Lambda}\left(\left\{K_{p}(U)\right\}\right)=\left(2^{-|\Lambda|} \prod_{p \in \Lambda} e^{K_{p}(U)}\right) \sum_{\Gamma \subset \Lambda} z_{\Gamma}(U) .
$$

The sum is over all sets $\Gamma$ of disjoint (compatible) contours $\left\{\zeta_{1}, \ldots, \zeta_{|\Gamma|}\right\}$ and

$$
z_{\Gamma}(U) \equiv \prod_{\zeta \in \Gamma} z_{\zeta}(U)
$$

with

$$
z_{\zeta}(U) \equiv \prod_{p \in \zeta} \exp \left(-2 K_{p}(U)\right)
$$

the activity of contour $\zeta$, and $K_{p}(U)$ given by (2.23). Noting that $\langle\gamma[C]\rangle_{Z(2)}\left(\left\{K_{p}(U)\right\}\right)=$ $\langle\gamma[S]\rangle_{Z(2)}\left(\left\{K_{p}(U)\right\}\right)$, where $\partial S=C$, it is easily seen that the expansion of the numerator

$$
Z_{Z(2), \Lambda}^{C}(U) \equiv Z_{Z(2), \Lambda}(U)\langle\gamma[C]\rangle_{Z(2)}\left(\left\{K_{p}(U)\right\}\right)
$$

is given by the expansion (3.17) after replacement of the contour activities $z_{\zeta}(U)$, eq. (3.19), by

$$
z_{\zeta}^{C}(U)=(-1)^{l(C, \zeta)} z_{\zeta}(U) \quad, \quad l(C, \zeta)=|S \cap \zeta| \quad(\bmod 2)
$$

(Here $l(C, \zeta)$ is the $(\bmod 2)$ linking number of the contour $\zeta$ with the loop $C$.)

Letting

$$
\mathcal{Z}_{Z(2), \Lambda}=\sum_{\Gamma \subset \Lambda} z_{\Gamma}(U), \quad \mathcal{Z}_{Z(2), \Lambda}^{C}=\sum_{\Gamma \subset \Lambda} z_{\Gamma}^{C}(U)
$$

and applying Jensen's inequality in (3.13) gives

$$
\begin{aligned}
W[C] & \geq \frac{1}{4} \int d \hat{\mu}_{\Lambda}(U) \exp \left(\ln \mathcal{Z}_{Z(2), \Lambda}^{C}(U)-\ln \mathcal{Z}_{Z(2), \Lambda}(U)\right) \\
& \geq \frac{1}{4} \exp \left(\int d \hat{\mu}_{\Lambda}(U)\left(\ln \mathcal{Z}_{Z(2), \Lambda}^{C}(U)-\ln \mathcal{Z}_{Z(2), \Lambda}(U)\right)\right) \\
& \equiv \frac{1}{4} \exp \mathcal{F}(\beta, \lambda) .
\end{aligned}
$$

Noting that $\mathcal{Z}_{Z(2), \Lambda}(U)=1$ at $z_{\zeta}(U)=0$, we may define $\ln \mathcal{Z}_{Z(2), \Lambda}(U)$ as that continuous branch of the logarithm which vanishes at vanishing contour activity, and similarly for $\ln \mathcal{Z}_{Z(2), \Lambda}^{C}(U)$. A closed form representation of $\mathcal{F}(\beta, \lambda)$ is given by the Möbius inversion representation of $\ln \mathcal{Z}_{Z(2), \Lambda}(U)$ and of $\ln \mathcal{Z}_{Z(2), \Lambda}^{C}(U)$ as a finite series (for finite $\Lambda$ ) of 
logarithms of partition function of linked clusters of single multiplicity [12]. Expanding these logarithms leads to the standard expansion of linked clusters of repeated multiplicities:

$$
\mathcal{F}(\beta, \lambda)=\int d \hat{\mu}_{\Lambda}(U)\left(\sum_{Q \subset \Lambda} a(Q)\left(z_{Q}^{C}(U)-z_{Q}(U)\right)\right)
$$

The sum is over all linked clusters $Q=\left\{\zeta_{1}, \ldots, \zeta_{n_{Q}}\right\}$ of contours with the property that at least one contour in each $Q$ winds around $C$. Multiple copies of a contour are allowed to appear as distinct members in a cluster, and

$$
a(Q)=\sum_{G(Q)}(-1)^{|G(Q)|}
$$

Here the sum is over all connected graphs $G(Q)$ on the vertex set $\left\{\zeta, \ldots, \zeta_{n_{Q}}\right\}$ with a line connecting two vertices if they represent incompatible (not disjoint) contours. $|G(Q)|$ denotes the number of lines in $G(Q)$.

The expansion in (3.23) converges for sufficiently small activities $z_{\zeta}(U)$. Let $\left|z_{\zeta}(U)\right| \leq$ $\exp (-b|\zeta|)$. The number of contours of size $q$ with one plaquette fixed is bounded by $[10(d-2)]^{10(d-2) q}$. Applying well-known results on the convergence of the polymertype cluster expansion [13], [14], it follows that the expansion on the r.h.s. of (3.23) is absolutely convergent, uniformly in $|\Lambda|$ and over $\Omega_{\Lambda}$, provided

$$
b \geq 10(d-2) \ln 10(d-2)+\ln 5 \quad ;
$$

hence, by (2.23), $\lambda$ sufficiently large. Uniform convergence allows integration term by term, so we have

$$
\mathcal{F}(\beta, \lambda)=\sum_{Q \subset \Lambda} a(Q)\left(\left\langle z_{Q}^{C}(U)\right\rangle-\left\langle z_{Q}(U)\right\rangle\right)
$$

We now observe that, for $\beta$ sufficiently large, the series (3.26) converges for all $\lambda \geq 0$. To show this, we bound $\left\langle z_{Q}(U)\right\rangle$ uniformly in $|\Lambda|$ by chessboard estimates 15 . One finds (Appendix)

$$
\left\langle z_{Q}(U)\right\rangle \leq \prod_{\zeta \in Q} \hat{z}(\beta, \lambda)^{|\zeta|}
$$

where

$$
\hat{z}(\beta, \lambda)=c_{1} e^{-c_{2} \beta-2 \lambda}
$$

with positive constants $c_{1}(d), c_{2}(d)$ depending only on the dimension $d$. It follows that (3.26) converges absolutely and uniformly in $|\Lambda|$, provided $|\hat{z}(\beta, \lambda)| \leq e^{-b}$ with $b$ satisfying (3.25); hence for $\beta$ large enough, and all $\lambda \geq 0$. Uniqueness of analytic continuation then implies that the representation of $\mathcal{F}$ by the cluster expansion in (3.26), originaly obtained in the domain of large $\operatorname{Re} \lambda$, is valid in this extented convergence domain $\operatorname{Re} \lambda \geq 0$. 
The leading contribution to (3.26) comes from the shortest possible contours, each consisting of $2(d-1)$ plaquettes forming the coboundary of a bond on the loop $C$, and there are $|C|$ of them. A bound on the remainder by the same as leading $\mathcal{O}(|C|)$-type behavior is a standard corollary of the polymer expansion convergence proof (e.g. [10]). This concludes the proof of the Theorem 3.1 for the action (2.9).

\section{A Appendix}

Let $\Pi_{e}$ denote the set of 'even' 2-dimensional $[\kappa \lambda]$-planes: $x^{\mu}=2 n^{\mu}$, integer $n^{\mu}, \mu \neq$ $\kappa, \lambda$. If $\left|\Pi_{e}\right|$ and $|\Lambda|$ denote the number of plaquettes in $\Pi_{e}$ and $\Lambda$, respectively, we have $\left|\Pi_{e}\right|=|\Lambda| / s$, where $s=2^{d-3} d(d-1)$. Consider now a cluster $Q$, and let $\zeta_{e} \equiv \zeta \cap \Pi_{e}$. Using RP to reflect repeatedly in $(d-1)$-dimensional planes with sites $x^{\mu}=\left(2 n^{\mu}+1\right)$, $\mu \neq \kappa, \lambda ; x^{\mu}=n^{\mu}, \mu=\kappa, \lambda$, and the fact that $\exp \left(-2 \beta\left|\operatorname{tr} U_{p}\right|\right) \leq 1$, one straightforwarly obtains the chessboard estimate

$$
\begin{aligned}
\left\langle z_{Q}(U)\right\rangle & =e^{-2 \lambda|Q|}\left\langle\prod_{\zeta \in Q} \prod_{p \in \zeta} e^{-2 \beta\left|\operatorname{tr} U_{p}\right|}\right\rangle \\
& \leq e^{-2 \lambda|Q|}\left\langle\prod_{\zeta \in Q} \prod_{p \in \zeta_{e}} e^{-2 \beta\left|\operatorname{tr} U_{p}\right|}\right\rangle \\
& \leq e^{-2 \lambda|Q|}\left\langle\prod_{p \in \Pi_{e}} e^{-2 \beta\left|\operatorname{tr} U_{p}\right|}\right\rangle^{\left|Q_{e}\right| / \Pi_{e} \mid},
\end{aligned}
$$

where $|Q|=\sum_{\zeta \in Q}|\zeta|$, and $\left|Q_{e}\right|=\sum_{\zeta \in Q}\left|\zeta_{e}\right|$. To estimate the last expectation on the r.h.s. in (A.1) it now suffices to bound the numerator from above by its maximum :

$$
\left\langle\prod_{p \in \Pi_{e}} e^{-2 \beta\left|\operatorname{tr} U_{p}\right|}\right\rangle \leq Z_{\Lambda}^{-1} e^{\lambda|\Lambda|} e^{2 \beta\left(|\Lambda|-\left|\Pi_{e}\right|\right)} .
$$

The partition function in the denominator is bounded from below by restricting $\gamma_{b}$ to 1 , and each $U_{b}$ integration to a small neighborhood of the identity such that $\left|\operatorname{tr} U_{p}\right| \geq 2 e^{-\delta}$ for all $p$. Let $\tau_{\delta}$ be the volume of this neighborhood. The constraints in $d \hat{\nu}_{\Lambda}(U)$ are then automatically satisfied, and we have

$$
Z_{\Lambda} \geq\left(\left(\frac{\tau_{\delta}}{2}\right)^{2 /(d-1)} e^{2 \beta e^{-\delta}+\lambda}\right)^{|\Lambda|}
$$

Now, given a cluster $Q$, we are free to pick the definition】 of the 'even' planes $\Pi_{e}$ so that $\left|\zeta_{e}\right| \geq|\zeta| / s$, i.e $\left|Q_{e}\right| \geq|Q| / s=|Q| / 2^{(d-3)} d(d-1)$. Combining this with (A.2), (A.3), we find

$$
\left\langle\prod_{p \in \Pi_{e}} e^{-2 \beta\left|\operatorname{tr} U_{p}\right|}\right\rangle^{\left|Q_{e}\right| /\left|\Pi_{e}\right|} \leq \hat{a}(\beta)^{|Q|}
$$

\footnotetext{
${ }^{7}$ We are free to choose 'even' coordinates (choice of lattice coordinate origin) and directions $[\kappa \lambda]$.
} 
where we defined

$$
\hat{a} \equiv \min _{\delta}\left(\left(\frac{2}{\tau_{\delta}}\right)^{2 /(d-1)} \exp \left(-\frac{2 \beta}{s}\left[1-s\left(1-e^{-\delta}\right)\right]\right)\right) .
$$

Thus $\hat{a}(\beta) \rightarrow 0$ exponentially as $\beta \rightarrow \infty$. (A.1) with (A.4) and

$$
\hat{z}(\beta, \lambda)=e^{-2 \lambda} \hat{a}(\beta)
$$

then produces the bound (3.27).

A more refined chessboard estimate, utilizing reflections onto every $d$-cell (dual site) of the lattice is possible (cp [4]), and results into elimination of the factor $1 / \mathrm{s}$ multiplying $\beta$ in the exponent in (A.5), a substantial numerical improvement. The simpler estimate given here, however, suffices for our purposes. 


\section{References}

[1] Davies, C.T.H., et al (eds): Lattice 97, Proceedings of the XVth International Symposium on Lattice Field Theory (Edinburgh). Nucl. Phys. B (Proc. Suppl.) 63 (1998)

[2] Kovács, T.G., Tomboulis, E.T.: Phys. Rev. D57, 4054 (1998); Nucl. Phys. B (Proc. Suppl.) 63, 534 (1998); ibid 53, 509 (1997)

[3] Del Debbio, L., Faber, M., Greensite, J., Olejnic, S.: Phys. Rev. D55, 2298 (1997); Nucl. Phys. B (Proc. Suppl.) 63, 552 (1998)

Del Debbio, L., et al: hep-lat/9801027 (1998)

[4] Yaffe, L.G.: Phys. Rev. D21, 1574 (1980)

[5] Tomboulis, E.T., Yaffe, L.G.: Commun. Math. Phys. 100, 313 (1985)

[6] Mack, G., Petkova, V.B.: Ann. Phys. (NY) 123, 442 (1979); ibid 125, 117 (1980); Z. Phys. C 12, 177 (1982).

[7] Tomboulis, E.T.: Phys. Rev. D 23, 2371 (1981)

[8] Tomboulis, E.T.: Phys. Lett. B, 303, 103, (1993)

[9] Griffiths, R.B.: J. Math. Phys. 8, 978 (1967)

Kelly, D.G., Sherman, S.: J. Math. Phys. 9, 466 (1968)

Ginibre, J.: Commun. Math. Phys. 16, 310 (1970)

[10] Seiler, E.: Gauge theories as a problem in Constructive Field Theory and Statistical Mechanics, Lecture Notes in Physics, vol. 159. New York: Springer-Verlag 1982

[11] Marra, R., Miracle-Sole, S.: Commun. Math. Phys. 67, 233 (1979)

[12] Koteckỳ, R., Preiss, D.: Commun. Math. Phys. 103, 491 (1986)

Münster, G.: Nucl. Phys. B180 [FS 2], 23 (1981)

[13] Brydges, D.: Critical phenomena, random systems, gauge theories - Les Houches 1984. Osterwalder, K., Stora, R. (eds). Elsevier Science Publishers 1986

[14] Cammarota, C.: Commun. Math. Phys. 85, 517 (1982)

[15] Frölich, J., Israel, R., Lieb, E., Simon, B.: Commun. Math. Phys. 62, 1 (1978)

Brydges, D., Frölich, J., Seiler, E.: Ann. Phys.(NY) 121227 (1979) 